\title{
The spectrum of tmLQCD with quark and link smearing
}

\author{
Abdou M. Abdel-Rehim* \\ Department of Physics, University of Regina, Regina, SK, S4S 0A2, Canada \\ and Department of Physics, Baylor University, Waco, TX, USA 76798-7316 \\ E-mail: Abdou_Abdel-Rehim@baylor.edu
}

\section{Randy Lewis, Robert G. Petry}

Department of Physics, University of Regina, Regina, SK, S4S OA2, Canada

\section{R. M. Woloshyn}

TRIUMF, 4004 Wesbrook Mall, Vancouver, BC, V6T 2A3, Canada

\begin{abstract}
The effect of using smeared sink operators on the hadron spectrum is studied for quenched twisted mass lattice QCD with up, down, and strange quarks. Gaussian smearing is used for quark fields, and stout link smearing for gauge fields. Smeared correlators are found to be dominated by the ground state with a small contribution from excited states, leading to an improved determination of some ground state masses.
\end{abstract}

XXIVth International Symposium on Lattice Field Theory

July 23-28, 2006

Tucson, Arizona, USA

${ }^{*}$ Speaker. 


\section{Introduction}

\subsection{Motivation}

Twisted mass lattice QCD (tmLQCD) offers an efficient mechanism for eliminating unphysical zero modes[1] and removing $O(a)$ errors from Wilson simulations[2]. An advantage of this formulation is that it allows simulations to be done at relatively light quark masses. Good results can be obtained for pions[3, 4] but the correlation functions for other hadrons tend to have significant statistical fluctuations when the quarks are light. In particular our previous exploration of the octet and decuplet baryons indicated that simple local operators are inadequate at small quark masses[5]. Here, we study whether the application of sink smearing can be useful in improving baryon signals and reducing statistical errors.

\subsection{Action}

We use the Wilson gauge action with a tmLQCD fermion action[6]

$$
S_{f}[\psi, \bar{\psi}, U]=a^{4} \sum_{x} \bar{\psi}(x)\left(M+i \mu \gamma_{5}+\gamma \cdot \nabla^{ \pm}-\frac{a}{2} \sum_{v} \nabla_{v}^{*} \nabla_{v}\right) \psi(x),
$$

where

$$
\begin{aligned}
\psi^{T} & =(u, d, c, s), \\
M & =\operatorname{diag}\left(M_{l}, M_{l}, M_{c}, M_{s}\right), \\
\mu & =\operatorname{diag}\left(\mu_{l},-\mu_{l}, \mu_{c},-\mu_{s}\right) .
\end{aligned}
$$

Forward $(\nabla)$, backward $\left(\nabla^{*}\right)$ and symmetric $\left(\nabla^{ \pm}\right)$derivatives are standard, and the $c$ field is irrelevant for our quenched simulations.

\subsection{Simulation details}

Simulation parameters are given in Table 1. These parameters correspond to maximal twist using the parity definition $[3,7,8]$. In the following, multi-state fits are used to extract ground state masses.

\section{Smearing Method}

Gaussian quark smearing is used at the sink[9],

$$
\begin{gathered}
q^{\mathrm{smr}}(x)=(1+\alpha \Delta)^{n_{\alpha}} q(x), \\
\Delta q(x)=\sum_{j= \pm 1, \pm 2, \pm 3}\left[U_{j}(x) q(x+\hat{j})-q(x)\right] .
\end{gathered}
$$

with stout spatial link variables[10]

$$
\begin{gathered}
U \rightarrow U^{(1)} \rightarrow U^{(2)} \rightarrow \ldots \rightarrow U^{\left(n_{\rho}\right)}, \\
U_{j}^{(n+1)}(x)=\exp \left(i \rho \Theta_{j}^{(n)}(x)\right) U_{j}^{(n)}(x) .
\end{gathered}
$$


The four smearing parameters were optimized for the reduction of excited state contributions at $\beta=6.0$, leading to $\alpha=0.15, n_{\alpha}=64, \rho=0.15$ and $n_{\rho}=16$. The link smearing parameters were optimized first using the static quark anti-quark potential (see Figure 1 and compare with [11]). A comparison between the effect of quark smearing and link smearing in reducing excited state contributions at our lightest and our heaviest masses is shown in Figure 2.

\section{Results}

\subsection{Vector Mesons}

In [12], results for the pseudoscalar and vector mesons with local operators were reported. A mass splitting between charged and neutral kaons was observed but, because of the large errors, no similar splitting between the neutral and charged vectors was found.

For any quark flavor $q$, the operators $\bar{q} \gamma_{j} q$ and $\bar{q} \sigma_{j 4} q$ both couple to the neutral vector meson, but the latter can mix with the axial vector away from maximal twist. In the present work, sink smearing was implemented for both of these operators, and the charged-neutral splitting was still insignificant at these lattice spacings. Representative effective mass plots are shown in the left panel of Figure 3.

Continuum extrapolations were performed linearly in $a^{2}$ for each quark mass. Subsequently, a simple linear fit in quark mass was used to approximate a chiral extrapolation, as shown in the right panel of Figure 3. Masses for the $\rho$ and $K^{*}$ that were obtained in this way are $O(10 \%)$ above experiment, as expected for a quenched simulation.

\subsection{Spin 1/2 baryons}

Source smearing allows for a more precise determination of ground state octet baryon masses. (For our previous exploration with local operators, see [5].) From the left panel of Figure 4, one sees the good scaling of the $\Sigma^{-}$mass with lattice spacing, while the $\Sigma^{+}$has a significant linear

\begin{tabular}{cccccl}
\hline$\beta$ & \#sites & \#configs & $a M$ & $a \mu$ & physical mass \\
\hline 5.85 & $20^{3} \times 40$ & 594 & -0.8965 & 0.0376 & $\sim m_{s}$ \\
& & & -0.9071 & 0.0188 & $\sim m_{s} / 2$ \\
& & & -0.9110 & 0.01252 & $\sim m_{s} / 3$ \\
6.0 & \multirow{2}{*}{$20^{3} \times 48$} & \multirow{2}{*}{600} & -0.8110 & 0.030 & $\sim m_{s}$ \\
& & & -0.8170 & 0.015 & $\sim m_{s} / 2$ \\
& & & -0.8195 & 0.010 & $\sim m_{s} / 3$ \\
6.2 & \multirow{2}{*}{$28^{3} \times 56$} & \multirow{2}{*}{200} & -0.8210 & 0.005 & $\sim m_{s} / 6$ \\
& & & -0.7367 & 0.021649 & $\sim m_{s}$ \\
& & & -0.7378 & 0.010825 & $\sim m_{s} / 2$ \\
& & & -0.7389 & 0.003616 & $\sim m_{s} / 3$ \\
& & & & & $\sim m_{s} / 6$ \\
\hline
\end{tabular}

Table 1: Simulation parameters. 


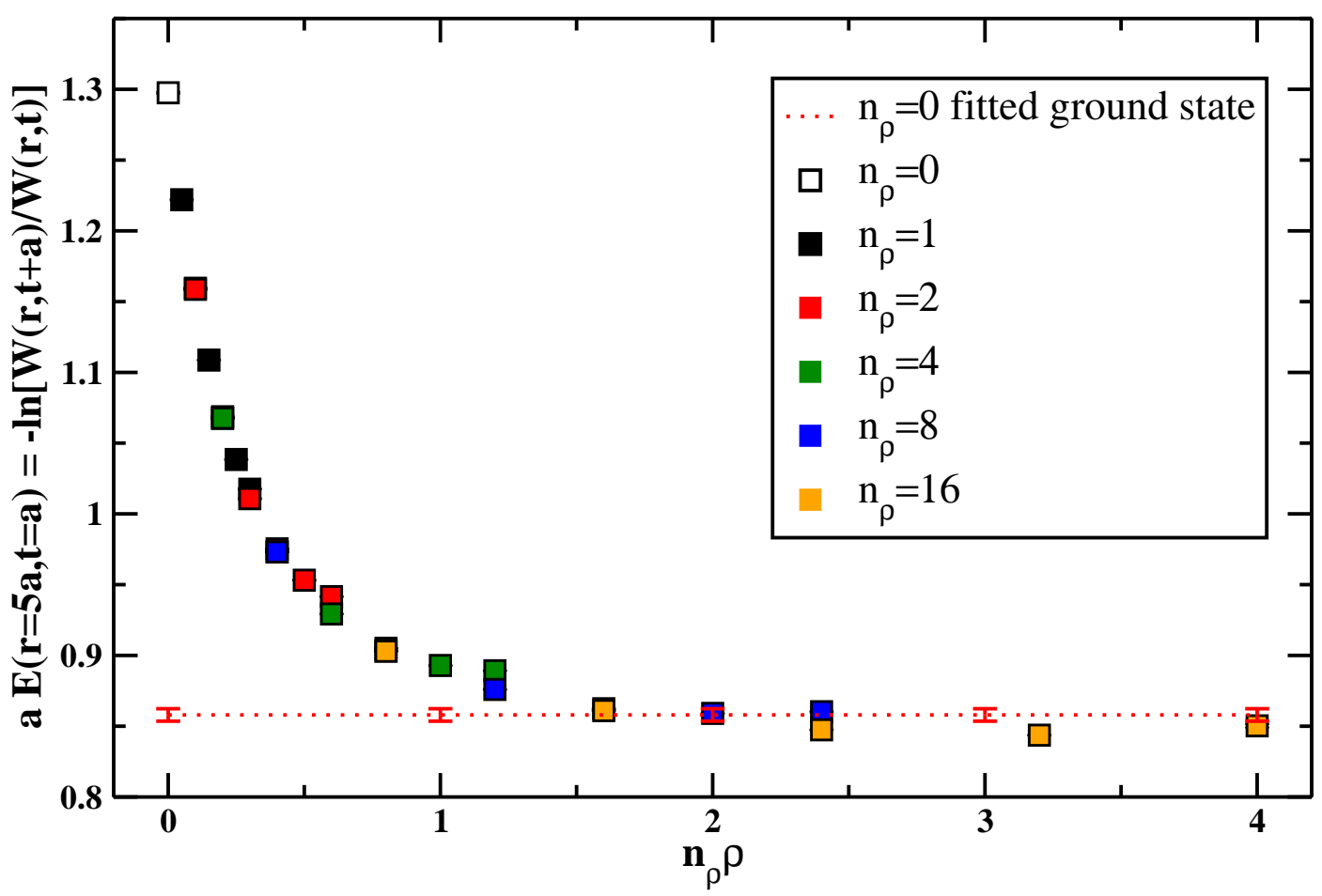

Figure 1: Effective mass for $\beta=6.0$ at temporal separation $\mathrm{t}=\mathrm{a}$ corresponding to the static quark-antiquark potential at spatial separation $\mathrm{r}=5 \mathrm{a}$, as a function of the stout link smearing parameters $\rho$ and $n_{\rho}$. The fitted ground state shown with no smearing used a two-exponential fit.

dependence on $a^{2}$. Since our tmLQCD corresponds to degenerate up and down quark masses, the observed splitting among $\Sigma^{ \pm}$masses at $\beta=5.85$ is a twist artifact. For $\beta \geq 6.0$, this artifact is not statistically significant. A similar twist artifact was observed among the $\Xi$ baryons.

Like the vector mesons, the quenched baryon masses obtained from simple linear fits in the quark mass are systematically larger than experiment, as evidenced by the right panel of Figure 4 . The ordering of $N, \Lambda, \Sigma$ and $\Xi$ masses is correct.

\subsection{Spin $3 / 2$ baryons}

Even with smearing, quarks much lighter than $m_{s}$ make fitting difficult. In Figure 5, the best case ( $\Omega$ has only strange valence quarks) and the worst case ( $\Delta$ has only light valence quarks) are shown. As expected, the quenched $\Omega$ is heavier than experiment. The $\Delta$ data are noisier than one would like; clearly sink smearing and/or additional operators should be explored in this context. Hints of twist artifacts can be seen at $\beta=5.85$, but the over-all poor quality of $\Delta(1232)$ data underscores the importance of future improvements like group-theoretical operators and source smearing.

\section{Discussion}

Sink smearing was effective in reducing excited state contributions. However, this did not 

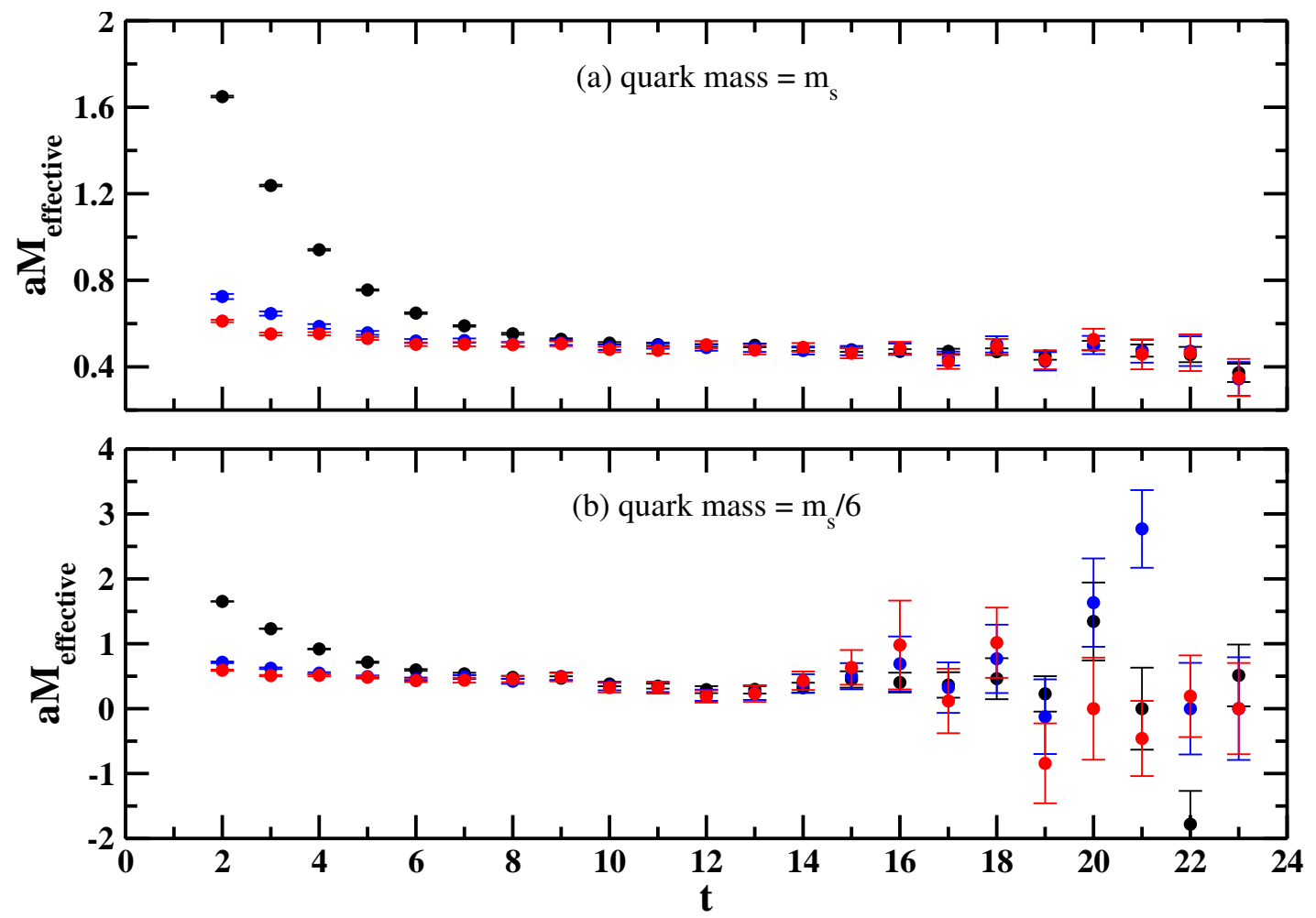

Figure 2: Effective mass plot for the charged vector at $\beta=6.0$ with the (a) heaviest and (b) lightest quark masses. Unsmeared results (black), quark smeared results (blue), and quark and link smeared results (red) are shown.
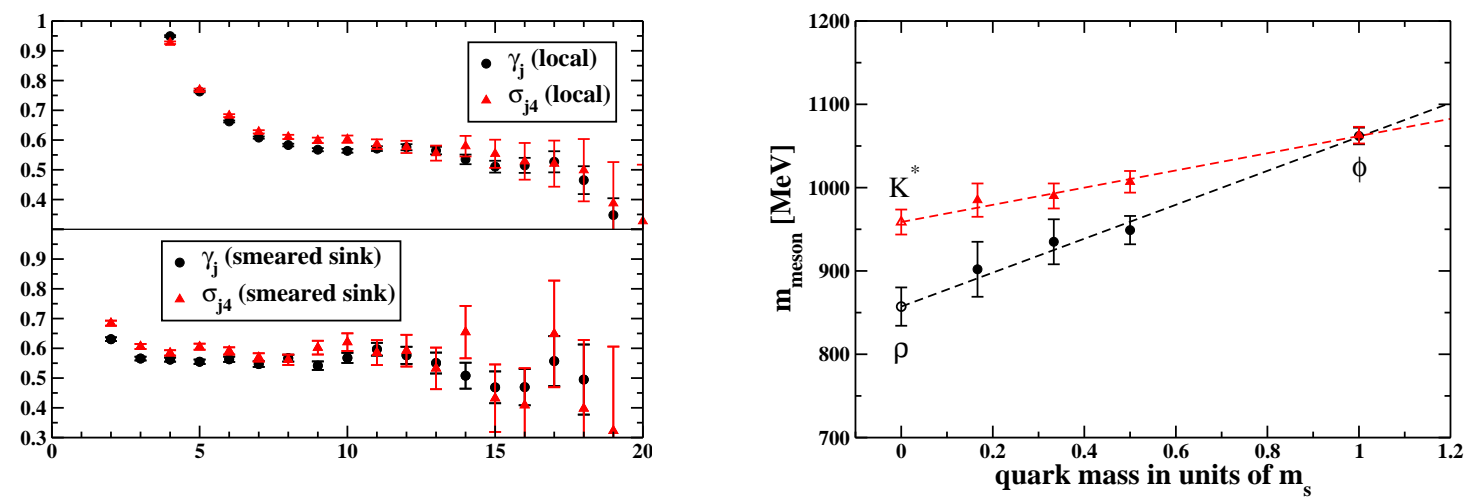

Figure 3: The left panel shows effective mass plots for the neutral vector meson with $\beta=5.85$ and quark mass $=m_{s} / 2$ for two local operators. The right panel shows simple linear fits to vector meson masses after continuum extrapolation. 

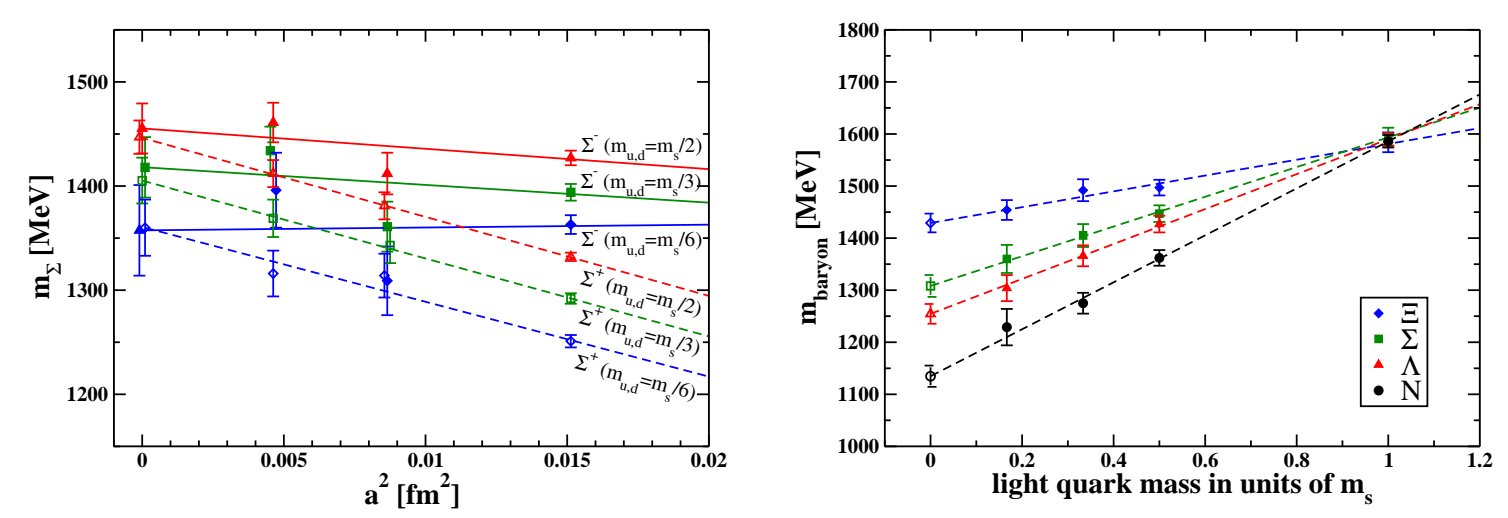

Figure 4: The left panel shows scaling of the $\Sigma^{+}$and $\Sigma^{-}$masses for three light quark masses, as well as linear extrapolations in $a^{2}$. Tiny horizontal offsets are for clarity. The right panel shows simple linear fits to the octet baryon masses, after continuum extrapolations.
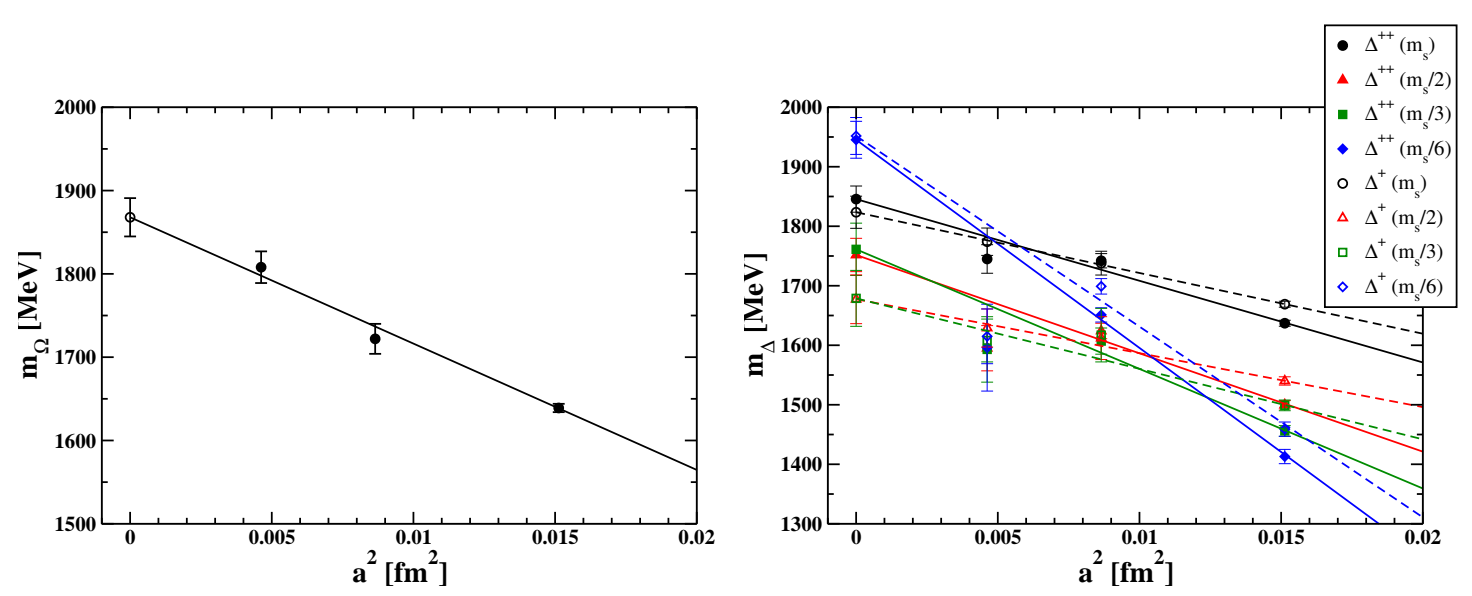

Figure 5: Scaling of the $\Omega$ and $\Delta$ masses. Linear fits to the $\Delta$ data are illustrative; note that $\beta=6.0$ and 6.2 scale within statistics. Tiny horizontal offsets are for clarity.

always lead to a more precise prediction for the ground state mass, compared with multi-state fits to unsmeared data. Nevertheless, some improvement in reducing systematic errors arising due to multi-exponential fits may have been realized.

Reasonably precise results for vector meson and spin 1/2 baryon masses were obtained, and indications of twisting artifacts were identified on our coarsest lattice. Even with smearing, the spin 3/2 data were of low quality for $m_{u, d}<m_{s} / 2$. Negative parity baryons and excited states were included in the multi-state fits, but were not useful for studies of the corresponding physical states and are not shown here.

Sink smearing is useful but not sufficient to allow precise studies of the baryon spectrum with tmLQCD. In future studies, source smearing should be considered. As well, operators constructed according to the lattice symmetries are being developed for tmLQCD[13]. 


\section{Acknowledgements}

This work was supported in part by the Natural Sciences and Engineering Research Council of Canada, the Canada Foundation for Innovation, the Canada Research Chairs Program and the Government of Saskatchewan.

\section{References}

[1] R. Frezzotti, P. A. Grassi, S. Sint and P. Weisz [Alpha collaboration], JHEP 0108, 058 (2001) [arXiv:hep-lat/0101001].

[2] R. Frezzotti and G. C. Rossi, JHEP 0408, 007 (2004) [arXiv:hep-lat/0306014].

[3] A. M. Abdel-Rehim, R. Lewis and R. M. Woloshyn, Phys. Rev. D 71, 094505 (2005) [arXiv:hep-lat/0503007].

[4] K. Jansen, M Papinutto, A. Shindler, C. Urbach and I. Wetzorke, Phys. Lett. B619, 184 (2005) [arXiv:hep-lat/0503031].

[5] A. M. Abdel-Rehim, R. Lewis and R. M. Woloshyn, PoS LAT2005, 032 (2006) [arXiv:hep-lat/0509056].

[6] C. Pena, S. Sint and A. Vladikas, JHEP 0409, 069 (2004) [arXiv:hep-lat/0405028].

[7] F. Farchioni et al., Nucl. Phys. Proc. Suppl. 140, 240 (2005) [arXiv:hep-lat/0409098].

[8] F. Farchioni et al., Eur. Phys. J. C 42, 73 (2005) [arXiv:hep-lat/0410031].

[9] M. G. Alford, T. Klassen and P. Lepage, Nucl. Phys. Proc. Suppl. 47, 370 (1996) [arXiv:hep-lat/9509087].

[10] C. Morningstar and M. J. Peardon, Phys. Rev. D 69, 054501 (2004) [arXiv:hep-lat/0311018].

[11] S. Basak et al., PoS LAT2005, 076 (2006) [arXiv:hep-lat/0509179].

[12] A. M. Abdel-Rehim, R. Lewis, R. M. Woloshyn and J. M. S. Wu, Phys. Rev. D 74, 014507 (2006) [arXiv:hep-lat/0601036].

[13] D. Harnett, R. Lewis and R. G. Petry, these proceedings [arXiv:hep-lat/0609071]. 